\title{
Modelo de competencias directivas para la autonomía de gestión y liderazgo en supervisores de educación básica en el marco de las reformas educativas en México
}

\section{Management and leadership autonomy model of supervisors of basic education in the framework of educational reforms in México}

\author{
Corona Velázquez, Luis ${ }^{1 *}$, Ramírez Elías, Gloria ${ }^{1}$ y Vázquez Fernández, Enrique ${ }^{1}$ \\ ${ }^{1}$ Universidad Autónoma de Tlaxcala \\ México \\ lcoronav@gmail.com
}

\begin{abstract}
Resumen
El trabajo de investigación es un avance de la tesis doctoral en Ciencias de la Administración; orientado a la construcción de un modelo propositivo de competencias directivas para la autonomía de gestión y liderazgo en el contexto de la supervisión escolar de educación básica en el contexto mexicano. El modelo propuesto se fundamenta sobre dos ejes epistemológicos: autonomía y liderazgo desde un enfoque de competencias. Resultado del debate de los modelos teóricos, se plantea la contemplación viva y un proceso de abstracción de los principios, características y regularidades de la autonomía de gestión y liderazgo para construir una realidad mejorada de las competencias directivas de supervisores escolares. Se estudia el contexto de las competencias directivas de supervisores escolares de una provincia de México en la perspectiva de la Reforma Educativa, destacando el principio de la escuela al centro, la autonomía de gestión, la ruta de mejora y los consejos técnicos. La metodología utilizada fue la cualitativa para construir los principios del modelo a través de entrevistas en profundidad y grupos focales que permitieron "captar" los significados y sentidos de la autonomía de gestión y liderazgo como competencias directivas, pero también se utilizaron elementos de la metodología cuantitativa para identificar las tendencias de competencias directivas a través de un instrumento de competencias intratégicas, estratégicas y de eficacia personal. El modelo propositivo de competencias directivas se estructura en una primera fase denominada fundamentación, una segunda fase es la definición de un perfil deseable de competencias directivas para la autonomía de gestión y liderazgo y la identificación de necesidades de formación y desarrollo de competencias directivas que permitan estructurar el sistema de formación; la tercera fase es la implementación estratégica del modelo con la planificación y evaluación del modelo.
\end{abstract}

Palabras clave: Modelo teórico, competencias directivas, autonomía de gestión, Liderazgo..

\begin{abstract}
The research work is an advance of the doctoral thesis in Administration Sciences; oriented towards the construction of a proactive model of managerial competences for the autonomy of management and leadership in the context of school supervision of basic education in the Mexican context. The proposed model is based on two epistemological axes: autonomy and leadership from a competence approach. The result of the debate of the theoretical models, live contemplation and a process of abstraction of the principles, characteristics and regularities of management autonomy and leadership to build an improved reality of the managerial competences of school supervisors. The context of the directive competences of school supervisors of a province of Mexico is studied in the perspective of the Educational Reform, emphasizing the principle of the school to the center, the autonomy of management, the route of improvement and the technical advice. The methodology used was qualitative to build the principles of the model through in-depth interviews and focus groups that allowed "capture" the meanings and meanings of management autonomy and leadership as managerial competencies, but also elements of the quantitative methodology were used to identify the tendencies of managerial competences through an instrument of intra-strategic, strategic and personal efficiency competencies. The proactive model of managerial competences is structured in a first phase called foundation, a second phase is the definition of a desirable profile of managerial competencies for the autonomy of management and leadership and the identification of training needs and development of managerial competences that allow structuring the training system; The third phase is the strategic implementation of the model with the planning and evaluation of the model.
\end{abstract}

Key words: Theoretical model, managerial competences, autonomy of management, Leadership.. 


\section{Introducción}

Las tendencias sobre estudios e investigaciones de competencias directivas en organizaciones escolares en América Latina se han incrementado en las tres últimas décadas a partir de las transformaciones tecnológicas y los procesos de globalización que demandan los sectores de la economía mundial; poniendo en tela de juicio los modelos tradicionales de educación que respondían a lo que (Bauman, 2007) denomina, el traspaso de la "modernidad sólida" a una "modernidad líquida" caracterizada por la incertidumbre, volatilidad y desechabilidad, en donde todo tipo de artefacto cultural, económico y político se diluye en turbulencias "líquidas". Es así que la construcción del modelo teórico propuesto demanda de principios vitales, con un carácter instituyente desde el imaginario social de la autonomía y liderazgo para la gestión de organizaciones escolares para la supervisión escolar.

A partir de la década de los años 60s surgen incipientemente lo conceptos de competencias laborales, e inciden como nuevos retos para la educación superior de formar profesionales con altas cualificaciones laborales que detonen el crecimiento y desarrollo organizacional fundamentalmente el industrial y empresarial como factores del desarrollo económico de las naciones, Mertens clasifica los modelos de competencias en estructurales y funcionales, como unidades productivas de la empresa. La gran variedad de modelos en la sociología, la psicología, la economía, filosofía, etc., inciden en "representar una realidad mejorada"; en los modelos directivos sobresale en los estudios de competencias directivas el de competencias estratégicas, intratégicas y de eficacia personal (Chinchilla y Cardona: 1999). Para identificar competencias estratégicas que hacen referencia a la capacidad estratégica del directivo en relación con el entorno externo para el logro de resultados; las intratégicas tienen la capacidad ejecutiva y liderazgo, orientadas a conseguir el compromiso de los trabajadores y el crecimiento de los empleados y la organización; las competencias de eficacia personal que tienen en cuenta los hábitos que favorecen la relación fluida del directivo con el entorno. A través de la técnica del grupo focal se analizaron las competencias de autonomía y liderazgo en cada contexto en particular.

Los esfuerzos de los sistemas educativos por elevar el nivel de competencias directivas de supervisores, inspectores y directores escolares es impostergable, por lo que nos planteamos la siguiente pregunta científica: ¿Cuáles son las características de un modelo que eleven las competencias directivas para la autonomía de gestión y liderazgo en supervisores escolares de educación básica? El objetivo general planteado es: Diseñar un modelo que eleve las competencias directivas para la autonomía de gestión y liderazgo en supervisores escolares de educación básica, en el contexto de la educación básica de la ciudad de Tlaxcala, México. El diagnóstico permite un acercamiento a la realidad de los niveles de competencias directivas de supervisores y directores del nivel de educación básica así como los resultados de los exámenes nacionales y locales de los alumnos que permitieron relacionar la función directiva en los procesos de aprendizaje de los alumnos a través de la autonomía de gestión y el liderazgo. Las limitaciones de orden epistemológico es la gran ambigüedad conceptual del término "competencias "y la "formación directiva de supervisión"; por un lado el sentido de la autonomía es concebida en los sistemas educativos desde la perspectiva del paradigma regulatorio, por lo que autonomía está acotada a lo que los niveles centrales permiten que asuman tareas desde la escuela. En las de orden metodológico es el grado de subjetividad y significaciones con gran predominio de la heteronomía instituida acerca de las experiencias situadas de autonomía de gestión y liderazgo.

La contribución para el campo académico consiste en un modelo teórico propositivo, que se sustenta en dos pilares de competencias directivas como lo son la autonomía de gestión y liderazgo desde una perspectiva de principios y regularidades sistematizados en contextos particulares.

\section{Marco contextual de la reforma educativa}

En México el movimiento de reforma educativa a transitado en forma similar a la de los países de la región latinoamericana, desde 1992 se inicia el Acuerdo Nacional para la Modernización de la Educación Básica (ANMEB), con procesos descentralizadores, que en realidad eran desconcentradores; en febrero de 2012 promulgada por el poder legislativo federal; La reforma educativa plantea la necesidad de contar con personal docente calificado, preparado y competitivo. Por tal motivo, se realizó una modificación constitucional a la Fracción III del artículo Tercero, en el que incluye la creación del Servicio Profesional Docente, en la cual se establece que los logros y formación de los maestros, permitirá su pertenencia en el cargo y quienes aspiren a puestos de dirección y supervisión deben tener las cualidades requeridas y debe corresponderse al desempeño y mérito profesional. La reforma educativa también plantea la creación del Instituto Nacional para la Evaluación, para esto se adiciona la fracción IX al artículo Tercero. La finalidad es que éste Instituto tenga autonomía y capacidad técnica para evaluar al Sistema Educativo Nacional a nivel preescolar, primario, secundario y nivel medio superior. Además plantea la Autonomía de gestión en las escuelas, pretende un mejor uso de los recursos públicos en beneficio de los planteles, de tal forma que la comunidad podrá decidir cuáles son sus necesidades y en que se debe invertir los recursos. (Leyes Secundarias). Se contempla el fortalecimiento de la autonomía de gestión de las escuelas para resolver los problemas de operación básicos, con el fin de mejorar su infraestructura, comprar materiales educativos y resolver problemas de operación básicos, donde las instancias gubernamentales autorizarán a los directores de los planteles educativos (escuelas) para la resolución de estos retos. En el año 2017 surge el Nuevo Modelo Educativo. La autonomía de gestión escolar es un eje articulador de la reforma educativa en, el acuerdo 717 del diario oficial de 
la federación (DOF.07/03/2014) ${ }^{1}$. En el perfil, parámetros e indicadores para personal con funciones de dirección y supervisión (2016:129), asigna las funciones generales de un supervisor que impulsa el desarrollo profesional del personal docente y la autonomía de gestión de las escuelas; coordina, apoya y da seguimiento al trabajo del personal docente, directivo y de asesoría técnico pedagógica, y establece el enlace con las autoridades educativas para garantizar el aprendizaje de todos los alumnos. En este contexto de implicaciones y criticas al modelo educativo; presenta un rostro de una Reforma Laboral del magisterio y la de recuperar la rectoría de la educación por parte del estado mexicano; ante la gran influencia de los sindicatos de oposición y oficiales en pugna. En este sentido centramos el trabajo de las competencias directivas en un modelo de los principios y regularidades de la autonomía y liderazgo en supervisores escolares.

\section{Marco teórico}

El modelo es una abstracción que caracteriza el objeto; es una representación de la realidad perfeccionada donde se actúa; y al que se llega a partir de un proceso de aproximación gradual donde se transita de esa abstracción, al modelo concreto «esencial, generalizado y en su totalidad». Es una construcción teórica caracterizada por un nivel de abstracción, con base epistemológica y una base teórica que permiten las inferencias y la identificación de regularidades en el campo empírico. Considerando el planteamiento de(Bourdieu, Chamboredon, y Passeron, s.f.) quien afirma “... que es en los principios de su construcción y no en su grado de formalización que radica el valor explicativo de los modelos". En la ciencia, la formulación y fundamentación de principios revela el alcance de mayores niveles de esencialidad en el conocimiento teórico de los objetos y fenómenos. Los principios son: ... . ideas centrales, conceptos generales, punto de partida en la estructuración y exposición de la teoría científica, los que se convierten en la primera determinación de las ideas de una teoría y cumplen una función metodológica importante a la hora de explicar un nuevo conocimiento. Los principios pueden cumplir dicha función metodológica, al ser contentivos de reglas y/o precisar ciertas implicaciones que deben tenerse en cuenta al momento de su aplicación. Tienen, por consiguiente, una relevante importancia práctica al posibilitar que el conocimiento teórico sistematizado pueda ser utilizado en la optimización de los procesos que explican.

Sin duda, se puede designar por modelo cualquier sistema de relaciones entre propiedades seleccionadas, abstractas y simplificadas, construido conscientemente con fines de descripción, de explicación o previsión y, por

\footnotetext{
${ }^{1} \mathrm{Al}$ margen un sello con el Escudo Nacional, que dice: Estados Unidos Mexicanos.- Secretaría de Educación Pública. EMILIO CHUAYFFET CHEMOR, Secretario de Educación Pública, con fundamento en los artículos 3o. de la Constitución Política de los Estados Unidos Mexicanos; 2o., 3o., 6o., 8o., 12, fracción V Bis y 28 Bis de la Ley General de Educación; 5, fracción XVI, del Reglamento Interior de la Secretaría de Educación Pública. menciona "Que el fortalecimiento de la autonomía de gestión de las escuelas contribuirá a lograr sus meta nacional "México con educación de Calidad"; "garantizar la inclusión y la equidad en el SEN".
}

ello, plenamente manejable; pero a condición según Pierre Bourdieu “... de no emplear sinónimos de este término que den a entender que el modelo pueda ser, en este caso, otra cosa que una copia que actúa como pleonasmo con lo real y que cuando es obtenida por un simple procedimiento de ajuste o extrapolación, no conduce en modo alguno al principio de la realidad que imita (Bourdieu y cols., s.f.). En nuestro caso, el modelo ha requerido en su proyección de la necesaria revisión de las tendencias más generales que operan en el objeto de estudio, lo que de manera práctica nos ha permitido acercarnos a nuestra propuesta, el análisis crítico de la información teórica, así como el estudio de las competencias directivas pretéritas asociados a la formación de supervisores, de manera que se puedan identificar los principios y regularidades. Competencias directivas, de acuerdo a(Bourdieu y cols., s.f.) "es en los principios de su construcción y no en su grado de formalización que radica el valor explicativo de los modelos"(Bourdieu y cols., s.f.); así como las contradicciones fundamentales presentes en el objeto. La propuesta de un modelo teórico de competencias directivas en supervisores escolares deviene de la necesidad de fundamentar metodológicamente el proceso de investigación sobre la base de competencias directivas para la autonomía de gestión y el liderazgo como elementos hipotéticos de la investigación y diseño del modelo propuesto. Lo que permitirá que los supervisores escolares logren alcanzar niveles aceptables en el encargo social que cumplimentan las políticas educativas locales y nacionales en el actual entorno de las reformas educativas. Las colindanacias teóricas para el abordaje de un modelo de competencias directivas se estructuran a partir de perspectivas; sociológicas (Marx y Weber), psicológicas, de gestión, organizacionales y administrativas. Desde el enfoque por Competencias (Le Boterf: 2001; Boyatzis: 1982; José Moya: 2007; Chinchilla y Cardona: 1992) aluden a una relación dialéctica de conocimientos, habilidades y actitudes. La concepción de la autonomía (Castoriadis y Vicens, s.f.) y el liderazgo como competencias directivas en el campo de la gestión directiva escolar. (González; 2001). La concepción diferenciada del directivo y del docente en el modelo teórico propuesto para la formación de competencias (Koontz y Weihrich: 2008; Mace: 1990; Brito y Plasencia; 2011); Del Toro, Valiente y González: 2014; Fernández: 2002, Gómez: 2010; Valiente, González y Del Toro: 2013). La perspectiva epistemológica de la teoría organizacional (Chester Barnard; Etzioni; Scott; Nicos Mouzelis) con aportes positivistas, que para efectos del modelo propuesto no lo tomamos como referente; otro de los aportes de los estudios organizacionales desde una perspectiva crítica de la organización desde lo cualitativo, entendiendo a la organización como un fenómeno social, intenta conocer, entender y comprender a las organizaciones del comportamiento humano y no hacerlas eficaces como las teorías administrativas y de la organización positivistas; es decir se trata de poner a la organización al servicio del hombre. Entre los fundamentos de los estudios organizacionales se considera a la organización como una 
construcción empírica del sujeto; la organización se considera a la organización como objeto del sujeto; consideran a la organización como instrumento de la persona. El método es el interpretativo a través del método cualitativo, con una orientación eminentemente social; la anarquía organizada donde conviven el orden y el desorden; esto conlleva al cambio; la organización en los estudios organizacionales es ambigua y compleja; en los estudios organizacionales el tipo de organización se concentra en todo los tipos de organizaciones. El carácter de los estudios organizacionales son analíticos explicativos, propuestas de los estudios organizacionales son obtener el conocimiento a través de la investigación.

\section{Metodología}

El enfoque de la metodología cualitativa es el que orienta nuestro trabajo describiendo los elementos de reorganización de acontecimientos vitales y las diferentes reconstrucciones que dan sentido a las acciones de autonomía y liderazgo en la gestión escolar. Sin embargo la metodología cuantitativa nos allano el camino para encuestar 329 casos; para ello se utilizó el instrumento de Chinchilla y Cardona para identificar las competencias directivas; estratégicas, intratégicas y de eficacia personal con 30 reactivos, aplicado a 48 supervisores y 280 directores del nivel primario, validados a través del alfa de Cronbach. Las técnicas del grupo focal y las características de la entrevista semiestructurada para la exploración de sentidos y significados, se integraron en categorías a nivel experiencial personal e institucional para encontrar las características del perfil deseable de supervisores para elevar el nivel de competencias directivas en el campo de la autonomía de gestión y el liderazgo; se trabajaron dos sesiones de grupo focal con 10 supervisores y tres directores de escuela primaria de la localidad de Tlaxcala. Se estableció la estrategia analítica de datos cualitativos con la descripción, análisis e interpretación de significados.

El desarrollo metodológico para el estudio de las "Competencias Directivas" en supervisores y directores del nivel de primaria; se estructuro a partir de un diseño no experimental en virtud de que no se someten a control las variables relacionadas a las competencias directivas, es descriptivo, exploratorio y transversal, de acuerdo a los criterios de la metodología cuantitativa. La población de estudio son el total de supervisores y directores del nivel de primaria que laboran en la Secretaria de Educación Pública en el Estado de Tlaxcala del nivel federal con una cobertura en el Estado de Tlaxcala:

\begin{tabular}{|c|c|c|c|}
\hline Supervisores & Directores & Docentes & Alumnos \\
\hline 41 & 288 & 5239 & 142744 \\
\hline
\end{tabular}

A través de la técnica del cuestionario se estructuraron 30 ítems, que exploran tres dimensiones de competencias directivas, que se clasifican de la siguiente manera: Competencias estratégicas: Son aquellas necesarias para obtener buenos resultados de la organización escolar y entre estas están: la visión, la resolución de problemas, la gestión de recursos, la orientación al cliente y la red de relaciones efectivas.

Competencias intratégicas: Son aquellas necesarias para desarrollar a los empleados e incrementar su compromiso y confianza con la empresa, que según el referido modelo, se trata en esencia de la capacidad ejecutiva y de la capacidad de liderazgo, entre las cuales se mencionan: la comunicación, la empatía, la delegación, el coaching y el trabajo en equipo.

Competencias de eficacia personal, que son aquellos hábitos que facilitan una relación eficaz de la persona con su entorno. Estas competencias miden la capacidad de auto-dirección, la cual resulta imprescindible para dirigir a otros, potenciando de esta forma las competencias estratégicas e intratégicas.

Para medir el nivel de confiabilidad se piloteo el instrumento, utilizando la nomenclatura de Likert, con 5 opciones de respuesta se planteó el siguiente objetivo del instrumento:

Objetivo: identificar las competencias directivas que con mayor preferencia aplica en su contexto escolar.

Antigüedad en el cargo: $\quad$ Subsistema:

Escala Likert: Registre con una "X", la opción de su elección. (5)Totalmente de acuerdo, (4) De acuerdo. (3) Nivel medio. (2) en desacuerdo. (1) Totalmente en desacuerdo. Posteriormente al encontrar confiabilidad del instrumento con el Alfa de Cronbach, se aplicaron los 288 cuestionarios restantes, con un total de 330 casos que se ilustra en la tabla 1:

Tabla 1

Resumen del procesamiento de los casos

\begin{tabular}{|r|l|r|r|}
\hline \multicolumn{2}{|c|}{} & \multicolumn{1}{l|}{$\mathrm{N}$} & \multicolumn{1}{c|}{$\%$} \\
\hline \multirow{3}{*}{ Casos } & Validos & 328 & 99.4 \\
\cline { 3 - 4 } & Excluidos * & 2 & 0.6 \\
\cline { 3 - 4 } & Total & 330 & 100.0 \\
\hline
\end{tabular}

* Eliminación por lista basada en todas las variables del procedimiento.

Tabla 2

Estadísticos de fiabilidad

\begin{tabular}{|r|r|}
\hline Alfa de Cronbach & N de elementos \\
\hline .931 & 30 \\
\hline
\end{tabular}

El contraste en el modelo factorial es significativo (KMO-Meyer-Olkin) relaciona los coeficientes de correlación observadas en las variables; como se aprecia es cercano a UNO, lo que implica que la relación entre las variables es alta. 
Los coeficientes de correlación nos permite inferir que las competencias directivas determinadas, guardan una estrecha relación, por lo que favorece el planteamiento hipotético de que sean competencias que permitirán a los directores y supervisores escolares sean las que permitan consolidar y favorecer un modelo de formación y desarrollo de competencias para la función directiva.

De los componentes rotados, concluimos que las variables se agrupan en tres grandes grupo:

- Componente 1: la asociación de variables (valores remarcados) se agrupan a las competencias de eficacia personal, Autocritica, Autoconocimiento y aprendizaje personal.

- Componente 2: la asociación de variables (valores remarcados) se agrupan en las competencias intratégicas; gestión de recursos, Relaciones efectivas, Dirección de personas.

- Componente 3: la asociación de variables (valores remarcados) se encuentran dispersas entre las competencias de Iniciativa, Orientación interfuncional y cooperación interinstitucional, Visión del entorno.

Tabla 3

Matriz de transformación de las componentes

\begin{tabular}{|l|c|c|c|}
\hline Componente & 1 & 2 & 3 \\
\hline 1 & .713 & .690 & .122 \\
\hline 2 & -.687 & .723 & -.072 \\
\hline 3 & -.138 & -.032 & .990 \\
\hline
\end{tabular}

Método de extracción: Análisis de componentes principales. Método de rotación: Normalización Varimax con Káiser.

El desarrollo de la metodología cualitativa se orientó a través del grupo focal con diez supervisores del nivel de primaria; planteamos la utilización de un procedimiento metodológico que no sólo aluda a las experiencias, sino a la reorganización de los acontecimientos vitales; de acuerdo a Vela (2008:66) a la reconstrucción de sentidos, a la vinculación con los otros, a la reconstrucción de experiencias en donde el retorno a ellas, sea la recuperación de la experiencia propia; consideramos como criterio metodológico, la creación de sentidos cómo síntesis que facilitan a las personas, explicar, justificar y reconocer sus acciones como legitimas. Dando paso a las significaciones imaginarias de los supervisores escolares; se afirmaría que no sólo en los documentos, en las prácticas o las políticas, está todo dicho acerca de la supervisión escolar, ya que ubicándonos en la reconstrucción de imaginarios, la subjetividad de las personas siempre tiene algo que decir, que compartir, que reconstruir.

La técnica del grupo focal aplicado tuvo como objetivo el de reconstruir las significaciones imaginarias de los supervisores en relación a la autonomía de gestión y liderazgo construido en la dinámica de las organizaciones escolares, que se dan en un tiempo, lugar y contexto de escuelas primarias, ante la normatividad, las prácticas y códigos instituidos.

La información relevante o clave a obtener estuvo referida al campo subjetivo en donde se enmarca el imaginario de los supervisores a partir de expectativas, deseos, experiencias, significados y representaciones que impulsan el papel de supervisor, y la dinámica de sus competencias para la favorecer la autonomía de gestión y liderazgo.

\section{Resultados}

Derivado de los resultados cuantitativos orientados a las preferencias de competencias directivas para la autonomía de gestión; las competencias de eficacia personal o de aquellos atributos personológicos presentan menor preferencia o manejo, se refiere a los hábitos básicos de una persona con ella misma y con su entorno; el autocontrol. Manejo de estrés, capacidad de síntesis y toma de decisiones; revelan un menor porcentaje. De igual manera las competencias directivas estratégicas, que se refieren a la capacidad del directivo y a su relación con el entorno externo de las Organizaciones en donde la capacidad de reconocer y aprovechar, Reconoce y aprovecha las oportunidades, las amenazas y las fuerzas externas que repercuten en la organización que dirige. Desarrolla y mantiene una amplia red de relaciones con personas clave dentro de la organización y del sector. Entre otras. De manera signifcativa las competencias de mayor preferencia son las intratégicas, centrar la atención al interior de la dinámica organizacional, sobre todo en el marco normativo.

El modelo predominante en la función directiva de supervisores escolares de la presente investigación, es el modelo burocrático, las competencias directivas siguen el orden vertical de supervisión escolar; la autonomía en las encuestas presenta un rango muy por debajo de competencias directivas; el liderazgo es asumido en términos de un imaginario de "autoridad"; existe un exceso de rutinas y trámites burocráticos que absorben la tarea de gestión, con poca presencia en escuelas, en asesoramiento directivo y pedagógico y sometidos a la presión de los resultados de las pruebas estandarizadas nacionales y locales. La "formación" de competencias directivas sigue la tradicional capacitación, reuniones de equipos técnicos y una gestión con mayor fuerza en la planeación para abatir problemas de comprensión de lectura y habilidades matemáticas; por lo que el supervisor escolar queda alejado para el desarrollo de competencias directivas que favorezcan la intervención pedagógica.

En la reconstrucción de significaciones imaginarias, a través del grupo focal, los supervisores significan un doble papel en su función; el primero lo asumen como una autoridad que "inspecciona" en el antiguo modelo burocrático, en donde las competencias directivas en su normatividad vertical; está condicionada por la centralidad del sistema educativo en prácticas normativas y un sentido del panóptico controlado por el paradigma regulatorio, característico de los modelos clásicos del estado-nación. 
Po otro lado asumen roles de asesores pedagógicos en la perspectiva de su formación magisterial. Se ven sometidos a estas tensiones, en virtud de que la autoridad central, le demanda una visión y actuación gerencial, sin que en este campo se haya formado; entonces es una reproducción de competencias directivas de medios a, fines en el sentido de los esquemas burocráticos. Por lo tanto las competencias directivas siguen el sentido vertical, basado en la eficiencia y eficacia, reconociendo que es la norma, el medio más natural e idóneo para elevar el nivel de competencias directivas para la autonomía de gestión y liderazgo. Significan la función supervisora como una actividad directiva de autoridad, alejados de la función de asesoría pedagógica; ocasionalmente visitan escuelas de su zona escolar y casi nunca tienen contacto con profesores o alumnos en el salón de clase. La autonomía de gestión la "viven" o la experimentan reduciéndola a la aplicación de la guía de la "Ruta de Mejora", que es la metodología para transformar la escuela desde la propia escuela, así como las reuniones de los consejos técnicos escolares, que tienen establecidos procedimientos y técnicas para la participación social, pero que al carecer de competencias directivas para movilizar estas herramientas estratégicas del nivel central, se mecanizan las actividades y se dejan fuera elementos contextuales, complejos y ausencia de una sistematización para elaborar diagnósticos que orienten la flexibilidad curricular y la toma de decisiones para la gestión y desarrollo organizacional escolar.

\section{Conclusiones}

El modelo que se propone es el resultado de estas contradicciones y obstáculos de tradición burocrática hacia un modelo de competencias de autonomía y liderazgo, basado en principios, características y regularidades que fundamentan las dimensiones esenciales, la estrategia derivada de lo anterior conforma el sistema que permite elevar el nivel de competencias directivas, su implementación se encuentra en fase de desarrollo y su validación a través del método de experto.

\section{Referencias Bibliográficas}

Bauman, Z. (2007). Los retos de la educación en la modernidad líquida. Barcelona: Gedisa.

Boterf, G. (2001). Ingeniería de las competencias. Barcelona: Gestión 2000.

Bourdieu, P., Chamboredon, J.-C., y Passeron, J. C. (s.f.). El oficio de sociólogo: presupuestos epistemológicos. Siglo Veintiuno Editores Argentina. (OCLC: 52758294)

Cardona, P. (s.f.). En busca de las competencias directivas. IEEM: Revista de Antiguos Alumnos, 3(2), 84-89.

Castoriadis, C. (2002). Ciudadanos sin brújula. México: Coyocán.

Castoriadis, C., y Vicens, A. (s.f.). La institución imaginaria de la sociedad. Tusquets. (OCLC: 46305716) de Educación, L. G., y DE EDUCACION, L. G. (s.f.). Publicada en el diario oficial de la federación el 13 de julio de 1993. Última reforma, 13.

García-Lombardía, P., Cardona, P., y Chinchilla, M. N. (s.f.). Las competencias directivas mas valoradas. Ocasional Paper. IESE, Barcelona, España.

Habermas, J. (1998). Teoría de la acción comunicativa. Madrid: Taurus.

Hernández, R. (2006). Estadísticas con spss y metodología de la investigación. México: Trillas.

Kazmier, L. (2006). Estadísticas aplicada a administración y economía. México, D.F: McGraw-Hill.

Kerlinger, F. (1975). Investigación del comportamiento. México: Interamericana.

Leboyer, C. (1997). Gestión de las competencias : cómo analizarlas, cómo evaluarlas, cómo desarrollarlas. Barcelona: Gestión 2000.

Longo, F., y GIL, E. (s.f.). La dirección pública como aprendizaje: una experiencia de diseño y evaluación de la formación en gerencia pública. En IX congreso internacional del CLAD sobre la reforma del estado y de la administración pública.

Álvarez de Zayas, C. M. (s.f.). Epistemología de la educación.

Sampieri, R. (2006). Metología de la investigación. México: McGraw Hill.

Recibido: 4 de junio de 2017

Aceptado: 20 de septiembre de 2017 